\title{
MIMR PRESCRIBING PATTERN OF INTRAVENOUS ANTIBIOTICS FOR HOSPITALIZED PATIENT IN HOSPITAL JERANTUT
}

\author{
Yamni Vijayasamy ${ }^{1 *}$, Erma Wany Abdul Basit $^{2}$, Sumaiyah Abdul Aziz ${ }^{3}$ \\ ${ }^{1}$ Hospital Jerantut, Pahang, Malaysia \\ ${ }^{2} P K D$ Jerantut, Pahang, Malaysia \\ ${ }^{3}$ Hospital Sultan Haji Ahmad Shah, Pahang, Malaysia \\ *Corresponding Author's Email: yamni_v@hotmail.com
}

\begin{abstract}
Hospitalized patients are often prescribed with antibiotics and it can be a challenge for the prescriber. This study is conducted to review the prescribing pattern of intravenous antibiotics for hospitalized patients in Jerantut Hospital in accordance to the National Antibiotic Guideline and Antimicrobial Stewardship Program. Methods: It is a retrospective study which is conducted in Jerantut Hospital for six months. Data is collected by using hospital's IV Antibiotic Form. 377 forms are then chosen using simple random sampling method and analyzed by SPSS using Pearson Chi Square test. Results: The most utilized antibiotic group is Penicillin $(\mathrm{n}=256,67.9 \%)$ whereas IV Amoxicillin-Clavulanate $(\mathrm{n}=183,48.5 \%)$ being the most frequent antibiotic used in Jerantut Hospital. Study shows prescribing pattern has high adherence towards NAG ( $\mathrm{n}=349,92.8 \%)$ but poor compliance towards 72 hours review $(n=51,13.5 \%)$. There is no significant difference in prescribing pattern between different classes of IV antibiotics $(p=0.207)$. Conclusion: Penicillin is the most utilized antibiotic group and IV Amoxicillin-Clavulanate is the most frequently used antibiotic in Jerantut Hospital. This study proves practices in Jerantut Hospital is highly adhered towards NAG but poor compliance towards 72 hours review Further work should be carried out to investigate some of the possible reasons for this matter. Strategies to improve prescribing pattern would need cooperation from all healthcare providers.
\end{abstract}

Keywords: Prescribing Pattern; Intravenous Antibiotics; Antibiotic Utilization; Guideline Adherence

\section{INTRODUCTION}

World Health Organization (WHO) has been pressing on antibiotics resistance becoming the biggest threat to global health and development to the modern day. The number of resistant bacteria is increasing steadily while hospitalized patients are continuously prescribed with antibiotics (Remesh et al., 2013; Fernandes, 2006; Andrajati, Vlček \& Wahyudin,2004). Overuse and misuse are the main contributor to this followed by lack of new developments in the antibiotics filed ( $\mathrm{Ab}$ Rahman, Teng \& Sivasampu,2016; Nasr, Paravattil \& Wilby, 2017). A change in the prescribing pattern or conscious behaviour change is needed to reduce the resistance which has direct impact on longer hospitalized days, higher medical cost and increased mortality.

To help matters, National Antibiotic Guideline (NAG) produced by Ministry of Health (MOH) Malaysia aimed to provide guidance to healthcare personnel in the management of infections. Selection of antimicrobial agent can be challenging to the clinician thus, the guideline aligned with the rational use of medications defined by WHO which require patients to receive appropriate medications according to their clinical needs, right doses based on their health condition for an adequate time and cost that is lowest to them and their community.

\section{METHODOLOGY}

It is a retrospective study which is conducted in Hospital Jerantut, a 77-bed public hospital from January 2018 to June 2018. The study is approved and cleared by National Medical Research Register with ID NMRR-18-1047-40149.

This study involves a population size of 2238 with a sample size of 377 subjects using confidence level of $95 \%$ and margin error of $5 \%$. The sample size is calculated using website http://www.raosoft.com/ samplesize.html. Simple random sampling method will be used to select the subjects. Selection of even numbering from a randomized list of patient's names in Microsoft Excel will be performed.

Inclusion criteria are patients started on IV antibiotics and admitted warding. Exclusion criteria include pediatric and maternity patients, patient given stat IV antibiotic dose at emergency dose and stepdown patient for antibiotic continuation. In Hospital Jerantut, as a 
principle all practitioners have to fill in Intravenous Antibiotic Form (Appendix 1) upon starting patients on intravenous antibiotic.

The form provides necessary information such as patient's particulars, the chosen antibiotic, patient's diagnosis, dose and frequency of antibiotic. It also states the culture and sensitivity taken and includes 72 hours review column. This form will be used as a tool in our research, as it gives a clear and complete picture on the practitioners prescribing pattern. Throughout the data collection period, all completed forms will be collected and sorted according to inclusion and exclusion criteria. It will later be specifically coded into Microsoft Excel. Data analysis will be done using Microsoft Excel and SPSS.

The data will be analyzed by using Microsoft Excel and SPSS. Descriptive results will be expressed as frequency and percentages. Categorical data will be analyzed using Pearson Chi Square test. $P<0.05$ is considered statistically significant.

\section{RESULTS}

During six months of the study period, 377 IV Antibiotic Forms were chosen to be analysed. Penicillin class is the most frequently prescribed IV antibiotic $(\mathrm{n}=256,67.9 \%)$ followed by Cephalosporin $(\mathrm{n}=116,30.8 \%)$ and others $(\mathrm{n}=5,1.3 \%)$. Figure 1 shows that the most prescribed IV antibiotics across medical wards are IV AmoxicillinClavulanate $(n=183,48.5 \%)$, IV Ceftriaxone $(n=67$, $17.8 \%)$ and IV Cefuroxime ( $\mathrm{n}=35,9.3 \%)$. The highest number of infections reported are community acquired pneumonia (CAP) $(n=202,53.6 \%)$, followed by other type of infections $(\mathrm{n}=117,31.0 \%)$ consisting of urinary tract infection (UTI), diabetic foot ulcer (DFU), cellulitis and infected wound. Tropical infection includes leptospirosis and melioidosis are the least common type of infections found in Hospital Jerantut ( $\mathrm{n}=58,15.4 \%$ ). In C\&S testing, majority of samples taken are blood $(n=354,93.9 \%)$ while the others involve collection of sputum and urine $(\mathrm{n}=23,6.1 \%)$.

\section{Utilization of IV Antibiotics}

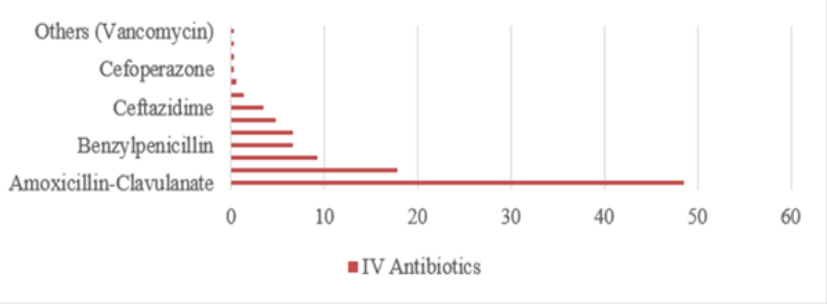

Figure 1: Utilization of IV Antibiotics
Most of the prescribed IV antibiotics adhere to NAG $(n=349,92.8 \%)$, this reflects the dose and frequency of prescribed IV antibiotics are in accordance with NAG. Result of IV antibiotic usage shows low compliance towards 72 hours review which implies no justification is recorded for antibiotics continuation after 3 days $(n=326,86.5 \%)$. Based on Pearson Chi Square Test performed, there is no significant difference in prescribing pattern between different class of IV antibiotics $(p=0.207)$. Hence, regardless of antibiotic classes, the NAG adherence and 72 hours review compliance are considered impartial, as prescribing pattern is determined by these two criteria. A form which is in compliance with both NAG and 72 hours review is considered as excellent prescribing pattern. If only one criterion is met, the prescribing pattern will be categorized as average while poor prescribing pattern means the prescribed IV antibiotics do not meet both criteria. The prescribing pattern in Jerantut Hospital leans towards average. The association between types of infection and the rational of sample taken for $C \& S$ test sampling are significant $(p<0.001)$. This finding proves that sample taken for $\mathrm{C} \& \mathrm{~S}$ testing depends on type of infection suspected.

\section{DISCUSSION}

Jerantut Hospital is a district hospital which takes part in cluster program. The main hospital that accommodates the clusters is Hospital Sultan Haji Ahmad Shah (HOSHAS). Apart from supplies and finance, their expertise is also shared. The presence of cluster and in-house doctors in Jerantut Hospital with different practices lead to variation in prescribing pattern. In this study, utilization of Intravenous (IV) antibiotic is evaluated. Based on the result, Amoxicillin-Clavulanate is the most utilized IV antibiotic $(n=185,48.5 \%)$ followed by Ceftriaxone $(n=$ $67,17.8 \%$ ) and Cefuroxime ( $\mathrm{n}=35,9.3 \%)$. AmoxicillinClavulanate is used to treat CAP which also a common infection found in Jerantut Hospital $(\mathrm{n}=202,53.6 \%)$ (Artoisenet et al., 2013). CAP is usually caused by gram positive, gram negative and atypical organism such as Streptococcus pneumonia, Haemophilus Influenza and Legionella sp. According to NAG, first line antibiotic treatment of CAP is IV Amoxicillin-Clavulanate $1.2 \mathrm{~g}$ TDS and the alternative is IV Ceftriaxone $1 \mathrm{~g}$ to $2 \mathrm{~g}$ OD for escalation upon failure of IV AmoxicillinClavulanate. Based on Malaysian Statistics on Medicines 2014 published by Pharmaceutical Services Division Ministry of Health Malaysia, Ceftriaxone is the most common third generation cephalosporin 
prescribed. (Masrahayu et al., 2006)

Apart from CAP, Ceftriaxone is also used to treat Leptospirosis which is caused by Leptospira $\mathrm{sp}$ (Disease control division department of public health ministry of health malaysia, 2011). Leptospirosis is a tropical infection which is currently re-emerging at an alarming state. There is a tremendous increase in the number of reported cases and frequency of outbreaks has been reported in Southeast Asia especially Thailand, Indonesia and Malaysia (Victoriano et al., 2009). In Malaysia, Pahang was listed as the $3^{\text {rd }}$ highest number of Leptospirosis incident with total amount of 184 out of 1418 cases in 2009 (Mendoza, 2010). This may be caused by the existence of large forest and establishment of felda area that lead to the contract of the disease. As stated in Asia Pacific Journal of Public Health, warm temperature and high humidity in Malaysia allow Leptospira sp to survive for long periods in the environment (Benacer et al., 2016).

Another common tropical infection in Pahang is Melioidosis. It has been reported that Pahang, as the active state in agriculture sector, is one of the hotspots for occurrence of Melioidosis where it had affected 6.1 per 100000 population per year from 2000-2003 (Nathan et al., 2018). Melioidosis is caused by Burkholderia pseudomallei, where patients with Diabetes Mellitus are more prone to be infected (How et al., 2005). Based on NAG and Pahang Guidelines for Management of Melioidosis, IV Ceftazidime is the first line treatment for melioidosis. This reflects the usage of Ceftazidime in this study $(\mathrm{n}=13,3.4 \%)$. Alternatively, Meropenem will be used to treat melioidosis in patients who do not respond to Ceftazidime. However, it is rarely used in this facility which makes it as a less common IV antibiotic $(n=2,0.5 \%)$.

Another antibiotic with low utilization in Jerantut Hospital is Piperacillin-Tazobactam $(n=5,6.6 \%)$. Both Meropenem and Piperacillin-Tazobactam are list A item which can only be prescribed by medical officers after consultation with specialists verbally or when visiting specialists are around. Furthermore, severe cases are referred to other tertiary hospital such as HOSHAS. In short, the most utilized IV antibiotic is Amoxicillin-Clavulanate followed by Ceftriaxone, Cefuroxime and Ceftazidime while the least common IV antibiotic utilized is Piperacillin-Tazobactam and Meropenem. This reflects on Penicillin $(n=256$, $67.9 \%$ ) being the most frequently prescribed antibiotic followed by Cephalosporin $(n=116,30.8 \%)$ and other classes $(n=5,1.3 \%)$.

An appropriate selection of antimicrobial therapy depends on several factors such as the knowledge of infectious disease, microbial cause of the infection and properties of the antimicrobials. The selection of therapy is usually complicated due to incomplete regimen in prior hospitalization causing resistance towards current bacteria (Ozgenç et al., 2011). Initial treatment for infections is often empiric and guided by clinical presentation. As a current basis in $\mathrm{MOH}, \mathrm{NAG}$ is used as a reference to guide doctors in prescribing appropriate antibiotics according to patients' condition. According to the data, most of the prescribed IV antibiotics adhere to NAG ( $\mathrm{n}=349,92.80 \%)$. Factors that contribute to high adherence rate includes experienced medical officers and nurses, specialist's involvement in prescribing list A antibiotics, participation of clinical pharmacists during ward rounds and availability of antibiotics. In addition, Jerantut Hospital is currently in the process of developing its own local antimicrobial guideline which is based on NAG. The local guideline will focus on common infections in Pahang and availability of antimicrobial agents in the hospital. This approach is encouraged by AMS program to guide healthcare providers in terms of appropriate use of antibiotic (Antimicrobial Stewardship, 2014).

On the other hand, the remaining of the prescribed IV antibiotics did not adhere towards NAG due to inappropriate choice of antibiotics or incorrect dose and frequency are prescribed $(n=28,7.20 \%)$. Most cases of non-adherence involve in the selection of IV antibiotic for the treatment of cellulitis. According to NAG, IV Cloxacillin is the preferred antibiotic to treat cellulitis. However, in this study, it is found that IV AmpicillinSulbactam is frequently prescribed for cellulitis which only is a choice, if the patient has diabetes mellitus (Lipsky et al., 2012). Lack of information in the IV antibiotic form such as comorbidities and age are limitations that contribute to the result in the study. Initiation of antibiotic should be justified according to patient's need before it is prescribed by following NAG and local guideline to avoid misuse and antibiotic resistance (Antimicrobial Stewardship, 2014). Overall, antibiotic selections in Jerantut Hospital are highly adhered towards NAG $(n=349,92.80 \%)$.

An antibiotic prescribed for longer than 72 hours has to be reviewed before it continues or make any changes towards the treatment regimen. In Jerantut Hospital, 
most of the prescribed IV antibiotics doesn't comply with the 72 hours review strategy $(n=326,86.5 \%)$. Involvement of cluster doctors who are unaware about the existence of IV antibiotic form might contribute to the result. Furthermore, antibiotic reviews are usually done verbally by the prescribers which make them overlook on the need to record their justification in the 72 hours review column. The implementation of 72 hours review in AMS program is important because it encourages prescribers to evaluate their decision on empirical antibiotic treatment and once C\&S results are available, a narrow spectrum antibiotic will be used.The importance of AMS program is also supported by WHO as it will provide positive impact on patient outcomes, including in-hospital mortality rates, reduce the length of stay, reduce treatment failure and reduce health care costs (WHO, 2018). Therefore, it is important to assess antibiotic use after 72 hours to provide the best course of action for patients.

Table 1: Association Between Prescribing Pattern and Classes of Antibiotics

\begin{tabular}{|c|c|c|c|c|}
\hline $\begin{array}{l}\text { Prescribing } \\
\text { Pattern }\end{array}$ & Poor & Average & Excellent & \\
\hline $\begin{array}{l}\text { Classes } \\
\text { of Antibiotics }\end{array}$ & $\mathrm{N}(\%)$ & $\mathrm{N}(\%)$ & $\mathrm{N}(\%)$ & $P$ value \\
\hline Cephalosporin & $10(8.5)$ & $88(74.6)$ & $20(16.9)$ & \multirow{3}{*}{0.207} \\
\hline Penicillin & $15(5.9)$ & $210(82.7)$ & $29(11.4)$ & \\
\hline Others & $1(20)$ & $4(80)$ & $0(0)$ & \\
\hline
\end{tabular}

Data was analysed using Fisher's Exact Test.

3 cells have (33.3\%) have expected count less than 5. The minimum expected count is .34

The study evaluates the relationship between different classes of IV antibiotics prescribed and prescribing pattern in Jerantut Hospital. However, there is no significant difference between the analysed group $(p=0.207)$. Based on the data in Table 1, the prescribing patterns are approximately the same across different classes of IV antibiotics. For instance, all antibiotic classes lean towards the average group. Hence, the result indicates that doctors in Hospital Jerantut do not have specific preference to follow NAG and to comply towards 72 hours review when different classes of IV antibiotics are prescribed (Quadranti et al., 2015). Furthermore, availability of higher and lower end antibiotics does not influence the type of prescribing pattern. As discussed earlier, involvement of cluster and in-house doctors play an important role in prescribing antibiotics. Prescribers must be familiarized with hospital practices particularly on the need to fill IV Antibiotic Form which has always been overlooked. This will help to improve compliance towards 72 hours review and NAG adherence which will lead to excellent prescribing pattern.

This study has taken the opportunity to analyse the association between types of infection and the rational of C\&S test sampling where the data shows that they are dependent with each other $(p<0.001)$. Majority of the analysed forms obtained appropriate culture based on patients' diagnosis $(n=360,95.5 \%)$. In Jerantut Hospital, blood sample is widely used to identify causative in CAP, tropical infections and others $(n=354,93.9 \%)(\operatorname{Lim}, 2011)$. However, it's not the only option as other samples such as sputum, swab and urine are also available. Blood $\mathrm{C} \& \mathrm{~S}$ is costly and require aseptic technique, which makes it more complex compared to others. The decision to start antibiotic often involve dilemma between uncertainty of infection and the risk of delaying treatment. Although sample is taken for C\&S test, the result is not available immediately. Generally, it requires at least 3 days in detecting the growth or presence of bacteria. If growth is detected, sample will be sent to HOSHAS to determine the type of organism and its sensitivity which will take about 5 days. Therefore, empirical antibiotic treatment can be given to the patients and deescalation of antibiotic therapy should be done when susceptible cultures from $C \& S$ test are available.

There are some steps to be taken to promote good prescribing practices among doctors. Ongoing medical education on antibiotic utilization is important to increase awareness on irrational use of antibiotics and its implications. During antibiotic awareness week in Jerantut Hospital, a few topics on antibiotic have been delivered to other healthcare providers to increase their knowledge on the appropriate use of antibiotic. In addition, AMS round has been initiated and the team will review antimicrobial prescribing and provide direct feedback to the prescribers.

\section{Limitation}

One of the limitations in this study is lack of information regarding patients' comorbidities where in some cases they significantly affect the choice of IV antibiotics prescribed such as in cellulitis and DFU. Apart from that, the reason of non-compliance towards 72 hours review is uncertain. It might be due to lack of awareness among doctors to complete the review or due to patient being discharged, where both former and 
later affect the result of this study.

\section{CONCLUSION}

In conclusion, the most utilized antibiotic class is Penicillin whereas IV Amoxicillin-Clavulanate being the most frequent antibiotic used in Jerantut Hospital. This study demonstrates high adherence towards NAG but poor compliance towards 72 hours review. Further study should be carried out to investigate some of the possible reasons for this matter. Strategies to improve prescribing pattern would need cooperation from all healthcare providers. On the other hand, the study found that types of infection significantly affect choice of sample taken for $\mathrm{C} \& S$ testing. A rational $C \& S$ test will help to improve antibiotic selection and therefore, reduce the unjustified use of antibiotics.

\section{Conflicts of Interest}

The authors declare that the research review was conducted in the absence of any commercial or economic associations that could be construed as a potential conflict of interest.

\section{ACKNOWLEDGEMENT}

Authors are grateful to the help extended by the management and staffs towards successful conduct and completion of this research.

\section{REFERENCES}

Ab Rahman, N., Teng, C. L., \& Sivasampu, S. (2016). Antibiotic prescribing in public and private practice: a cross-sectional study in primary care clinics in Malaysia. BMC Infectious Diseases, 16(1), 1-8.

Andrajati, R., Vlček, J., \& Wahyudin, I. (2004). Assessment of antibiotics use after introducing a hospital formulary by ATC/DDD methodology. Medical Journal of Indonesia, 13(3), 173-9.

Antimicrobial Stewardship (2014) Retrieve from: https://www.pharmacy.gov.my/v2/sites/default/fil es/document-upload/protocol-antimicrobialstewardship.pdf

Artoisenet, C., Ausselet, N., Delaere, B., \& Spinewine, A. (2013). Evaluation of the appropriateness of intravenous amoxicillin/clavulanate prescription in a teaching hospital. Acta Clinica Belgica, 68(2), 81-86.

Benacer, D., Thong, K. L., Verasahib, K. B., Galloway,
R. L., Hartskeerl, R. A., Lewis, J. W., \& Mohd Zain, S. N. (2016). Human leptospirosis in Malaysia: reviewing the challenges after 8 decades (19252012). Asia Pacific Journal of Public Health, 28(4), 290-302.

Disease control division department of public health ministry of health malaysia (2011). Guideline for the Diagnosis, Management, Prevention and Control of Leptospirosis in Malaysia. Retrieved from: https://www.moh.gov.my/moh/resources/ auto\%20download\%20images/589d71cb177d8.pdf

Fernandes, P. (2006). Antibacterial discovery and development - the failure of success?. Nature Biotechnology, 24(12), 1497-1503.

How, S. H., Ng, K. H., Jamalludin, A. R., Shah, A., \& Rathor, Y. (2005). Melioidosis in Pahang, Malaysia. The Medical Journal of Malaysia, 60(5), 606-613.

Lim, V. K. E. (2011). Leptospirosis: a re-emerging infection. The Malaysian Journal of Pathology, 33(1), 1 .

Lipsky, B. A., Berendt, A. R., Cornia, P. B., Pile, J. C., Peters, E. J., Armstrong, D. G., ... \& Senneville, E. (2012). 2012 Infectious Diseases Society of America clinical practice guideline for the diagnosis and treatment of diabetic foot infections. Clinical Infectious Diseases, 54(12), e132-e173.

Masrahayu, M., Ngau, Y.Y., Thong, K.S., Sameerah, S.A.R., Jacqueline, L., \& Rahela, A.K. (2006). Use of Antiinfectives. Malaysian Statistic on medicine 2006.Retrieved from: http://www.crc.gov.my/nhsi/ wp-content/uploads/publications/msom2006/ chapter15.pdf

Mendoza, R. L. (2010). Leptospirosis in the tropics: when prevention doesn't easily sell as a ton of cure. American Journal of Economics and Business Administration, 2(3), 307.

Nasr, Z., Paravattil, B., \& Wilby, K. J. (2017). The impact of antimicrobial stewardship strategies on antibiotic appropriateness and prescribing behaviours in selected countries in the Middle East: a systematic review. Eastern Mediterranean Health Journal, 23(6).

Nathan, S., Chieng, S., Kingsley, P. V., Mohan, A., Podin, Y., Ooi, M. H., ... \& How, S. H. (2018). Melioidosis in Malaysia: incidence, clinical 
challenges, and advances in understanding pathogenesis. Tropical Medicine and Infectious Disease, 3(1), 25.

Ozgenç, O., Genç, V. E., Ari, A. A., Sibel, E., Saçar, S., Ozunlu, H., ... \& Ergonul, O. (2011). Evaluation of the therapeutic use of antibiotics in Aegean Region hospitals of Turkey: a multicentric study. Indian Journal of Medical Microbiology, 29(2), 124.

Quadranti, N. R., Popović, B., Škrobonja, I., Skočibušić, N., \& Vlahović-Palčevski, V. (2015). Assessment of adherence to printed guidelines for antimicrobial drug use in a university hospital. European Journal of Hospital Pharmacy, 22(2), 113-117.
Remesh, A., Salim, S., Gayathri, A. M., Nair, U., \& Retnavally, K. G. (2013). Antibiotics prescribing pattern in the in-patient departments of a tertiary care hospital. Arch Pharma Pract, 4(2), 71.

Sample size calculator. Retrieved from: http://www. raosoft.com/samplesize.html.

Victoriano, A. F. B., Smythe, L. D., Gloriani-Barzaga, N., Cavinta, L. L., Kasai, T., Limpakarnjanarat, K., ... \& Adler, B. (2009). Leptospirosis in the Asia Pacific region. BMC Infectious Diseases, 9(1), 1-9.

WHO (2018), Retrieved from: http://www.who.int/ mediacentre/ factsheets/fs194/en/. 